\title{
L'offre de recherche converge-t-elle vers les besoins du secteur agroalimentaire au Cameroun? Une analyse par la bibliographie
}

\author{
Jules René Minkoua Nzié $e^{1,3, *}$ et Ludovic Temple ${ }^{2,4}$ \\ ${ }^{1}$ Université de Yaoundé II-Soa, Département d'Économie Publique, BP 1365, Yaoundé, Cameroun \\ 2 Cirad, Umr Innovation, B15, 73 rue JF Breton, 34398 Montpellier, France \\ ${ }^{3}$ University of Buea, Department of Economics and Management, PO Box 63, Buea, Cameroon \\ ${ }^{4}$ Univ Montpellier, Montpellier, France
}

\begin{abstract}
Résumé - Il est possible de connaître les impacts de la recherche en interrogeant dans la littérature la convergence entre la demande et l'offre de recherche dans les pays en développement où les statistiques sur l'innovation sont peu disponibles. Nous traitons cette interrogation dans le cadre du secteur agroalimentaire au Cameroun. La demande de recherche est estimée par les indicateurs de besoins élaborés à partir des opinions des chefs d'entreprises concernant le climat des affaires et la protection de l'environnement. L'offre de recherche est mesurée par un indicateur de pertinence des thématiques traitées dans les publications scientifiques faites par les auteurs camerounais. L'étude utilise les données bibliométriques sur la période 1991-2015, et celles du recensement général des entreprises de 2008. Elle constate une faible correspondance entre les besoins de développement des entreprises agroalimentaires et la littérature scientifique et discute les conditions d'usage de la méthode proposée.
\end{abstract}

Mots clés : recherche agronomique / offre et demande / environnement / affaires / Cameroun

\begin{abstract}
Does the supply of research converge towards the needs of the agri-food sector in Cameroon? A bibliographic analysis. Knowledge of the impacts of research can be done by querying in the literature the convergence between demand and supply of research in developing countries where innovation statistics are scarce. We are dealing with this question in the context of the agri-food sector in Cameroon. The demand for research is estimated by the indicators of needs developed from the opinions of business managers on the business climate and the protection of environment. The research supply is measured by a relevance indicator of the themes treated in scientific publications by Cameroonian authors. The study uses bibliometric data for the period 1991-2015, and the 2008 general census of enterprises. It notes a weak alignment between the development needs of agribusinesses and the scientific literature, and discusses the conditions of use of the proposed method.
\end{abstract}

Keywords: agricultural research / supply and demand / environment / business / Cameroon

\section{Introduction}

La demande pour une science qui réponde aux besoins sociétaux est de plus en plus forte chez les décideurs et les bailleurs publics et privés (Rafols et al., 2015). Cette demande interroge une offre de progrès scientifique et technologique susceptible d'induire des processus d'innovation connectés aux préoccupations sociétales. Dans les pays en développement, où les structures scientifiques, technologiques et industrielles sont relativement fragiles, les études tendent principalement à référencer les conditions d'existence de leurs

\footnotetext{
$\overline{\text { *Auteur de correspondance }}$ : minkouarene@yahoo.fr
}

interactions plutôt qu'à discuter de l'utilité de ces dernières (Gallaud, 2014; Casadella et Tahi, 2017). Une partie des difficultés à évaluer l'utilité de la science réside dans la faiblesse des bases de données statistiques et des compétences pour mesurer la capacité des politiques de recherche à stimuler les processus d'innovation intégrant les attentes de développement des divers acteurs. D'autre part, cette évaluation implique une démarche conceptuelle et empirique pour comprendre si et comment l'offre de connaissance scientifique s'accommode des besoins du secteur entrepreneurial, c'est-àdire de l'ensemble des acteurs et entreprises qui investissent dans l'innovation (Tiran et Uzunidis, 2017). En économie, la notion de besoin se définit comme une sensation de manque que l'on cherche à combler (Gueutin, 2012). Dans notre étude 
centrée sur le secteur agroalimentaire, le besoin porte sur l'identification des situations insatisfaisantes (manques), nécessitant une action correctrice potentielle. Nous proposons une démarche méthodologique mobilisant les données secondaires existantes pour analyser la convergence des priorités de recherche scientifique avec les enjeux de développement des entreprises du secteur agroalimentaire au Cameroun.

\subsection{Cadre conceptuel d'analyse des relations entre l'offre et la demande de science dans les pays en développement}

C'est avec un groupe d'auteurs transdisciplinaires du Center for Science and Technology Policy Research de l'Université du Colorado aux États-Unis, notamment Sarewitz et Pielke (2007) et McNie (2007), que les notions d'offre et de demande ont été utilisées pour appréhender les processus de prise de décision en matière scientifique. L'offre de science se conçoit en termes d'offre de connaissances et d'informations scientifiques utiles pour les différents acteurs (publics, privés, société civile) tandis que la demande, émanant de ces acteurs, correspond aux retombées sociétales issues de la mise en application des connaissances. Ces définitions diffèrent de celles de la micro-économie standard où l'offre de science s'appréhende comme le niveau de production scientifique associé à un niveau de dépense donné (Alston et al., 2010); et où la demande de science, quant à elle, s'apprécie comme le niveau de production scientifique désiré par les utilisateurs pour un niveau de dépense donné.

Pour Sarewitz et Pielke (2007), l'enjeu d'une conciliation entre l'offre et la demande de science consiste à établir des mécanismes institutionnels d'interaction permettant de produire une information et une connaissance utiles ou utilisables pour les preneurs de décision ou usagers, à l'instar des gestionnaires d'entreprise.

Une information est qualifiée d'utile si elle respecte trois critères : la pertinence, la crédibilité et la légitimité (McNie, 2007) (Tab. 1).

Notre étude se limitera à l'analyse de la pertinence comme mesure de l'utilité de l'information scientifique publiée. Nous faisons l'hypothèse que le référencement de l'information scientifique et technique par les revues à comité de lecture assure les critères de la crédibilité et de la légitimité. La prise en compte de ces critères (et plus loin dans l'article uniquement de la pertinence), détermine la confiance en l'information scientifique, nécessaire pour favoriser la formation des opinions, des attitudes ou des décisions auprès du public (Brewer et Ley, 2013).

Paradoxalement, dans les pays moins avancés (PMA), on trouve au niveau entrepreneurial une faible prise en compte des connaissances produites localement (Arocena et Sutz, 2010). Pour Reinert (2007), c'est probablement le reflet d'une spécialisation de ces pays vers des activités dépourvues de potentiel d'apprentissage industriel ou, pour d'autres auteurs (Rodrik, 2007; Arocena et Sutz, 2010), une faiblesse de la demande entrepreneuriale de connaissances qui rendrait l'offre locale de connaissances moins pertinente.

Les activités dépourvues de potentiel d'apprentissage, définies comme des activités non innovatrices, concernent, dans le cas des PMA, des entreprises qui n'engagent pas d'efforts innovants pour surmonter les obstacles liés à la
Tableau 1. Les critères d'une information scientifique utile. Table 1. Criteria of useful scientific information.

\begin{tabular}{ll}
\hline Critères & Significations \\
\hline Pertinence & $\begin{array}{l}\text { Sensibilité au contexte et prise en compte des } \\
\text { considérations écologiques, temporelles, } \\
\text { spatiales et administratives }\end{array}$ \\
Crédibilité & $\begin{array}{l}\text { Information perçue comme exacte, valide et de } \\
\text { grande qualité } \\
\text { Tégitimité }\end{array}$ \\
$\begin{array}{l}\text { diffuseurs d'information scientifique construite à } \\
\text { partir du capital social }\end{array}$ \\
\hline
\end{tabular}

Source: À partir de McNie (2007).

recherche de l'innovation (Sutz, 2012). Ces efforts, souvent technologiques, peuvent porter sur les défauts de qualité, les problèmes d'acquisition des matières premières, les problèmes d'équipements ainsi que de remplacement des pièces, le climat des affaires, ou les contraintes environnementales. Ces efforts engagent un processus d'apprentissage (Lundvall, 1992) structurant une demande entrepreneuriale de connaissances à travers deux mécanismes (Jensen et al., 2007, Hadhri et al., 2016) : celui de l'acquisition d'un savoir-faire par la pratique, l'usage et les interactions, d'une part, et celui de l'utilisation de savoirs codifiés, d'autre part. Quel que soit le mécanisme suivi, une corrélation institutionnelle forte entre offreurs et demandeurs de connaissances est nécessaire pour garantir l'utilité de la recherche scientifique comme instrument de développement économique.

\section{La contextualisation institutionnelle de la science au Cameroun}

Deux importantes études sur l'état de la science au Cameroun (Gaillard et Khelfaoui, 2000 ; Bisson et al., 2014) permettent d'identifier deux mouvements: un dynamisme, mais contraint, de l'offre de science, d'une part, et une relative faible expression de la demande de science, d'autre part.

\subsection{Le dynamisme de l'offre de science est fortement contraint}

L'activité scientifique au Cameroun commence à l'ère coloniale. Une phase d'institutionnalisation sera amorcée dès le milieu des années 1930 avec la création de la Société d'études camerounaises (SECAM) et des investissements publics dans la recherche qui ne cesseront de croître.

Après l'Indépendance, sont mis en place l'Institut de la recherche agronomique (IRA) et l'Institut de la recherche zootechnique (IRZ).

À partir du milieu des années 1980, une crise économique survient et affecte considérablement les moyens accordés au secteur de la recherche au Cameroun. Ainsi, l'offre de recherche est fortement contrainte par l'insuffisance des moyens financiers, mais également par la faiblesse des infrastructures numériques, qui rend difficile l'accès aux bases bibliographiques mondiales. Cette faiblesse des moyens rend les chercheurs nationaux dépendants des sources de 
financement étrangères, provoquant ainsi l'adhésion à des programmes de recherche parfois déconnectés des besoins de connaissance du secteur productif, et notamment du secteur agroalimentaire (Fofiri et al., 2015, Temple et al., 2017).

\subsection{La faible expression de la demande de science}

$\mathrm{Au}$ Cameroun, la demande formelle de science est d'abord institutionnelle, c'est-à-dire émanant historiquement des instances gouvernementales, coloniales puis nationales, qui exercent leur tutelle sur les institutions de recherche, constituant ainsi les premiers «utilisateurs des résultats de la recherche». Après l'Indépendance, le gouvernement camerounais accorde la priorité au secteur agricole et rural, comme base de développement économique. De nombreuses sociétés agro-industrielles sont créées sur fonds public durant cette phase de planification, pour les filières riz, banane, huile de palme, hévéa, coton, thé, maïs, cacao et café. Mais depuis les années 1990, les interactions entre la recherche publique et les entreprises se limitent aux recours ponctuels à l'expertise de la recherche publique et à quelques situations de collaboration par contrat (SODECOTON, Cameroon Development Corporation...).

\section{Cadre méthodologique d'analyse de la convergence entre offre et demande de recherche}

Nous questionnons ici le processus d'ajustement entre l'offre et la demande de recherche dans le contexte institutionnel d'un pays à revenus intermédiaires (PRI). Dans ce contexte, le nombre de brevets, classiquement utilisé dans les pays de l'Organisation pour la coopération et le développement économique (OCDE), est peu pertinent au regard du caractère émergent des institutions qui stabilisent les droits de propriété intellectuelle (Bisson et al., 2014). Dans les pays en développement, les nouvelles technologies de l'information permettent, depuis plus d'une décennie, d'accélérer la numérisation et la mondialisation de l'accès à la connaissance scientifique (Mouton et al., 2015). Cette numérisation est à la base de la présente étude bibliométrique sur l'analyse des mécanismes de convergence entre l'offre et la demande de recherche.

L'offre de recherche est mesurée par des indicateurs de pertinence des thématiques traitées dans les publications scientifiques dans le domaine agroalimentaire et environnemental $(E 2 A)$ par les universités et les institutions de recherche camerounaises et internationales. Ces indicateurs de pertinence caractérisent le contexte des acteurs (McNie, 2007) marqué par les principales thématiques qui polarisent l'actualité des contraintes des entreprises agroalimentaires camerounaises. Ces thématiques sont relatives au climat des affaires et à la protection de l'environnement. Les indicateurs de pertinence associés sont calculés par VOSviewer qui génère le nombre d'apparitions des termes ou thématiques répertoriés dans les publications scientifiques référencées sur la période analysée. L'analyse bibliométrique s'est limitée aux titres et aux résumés des publications.
La demande de recherche est approchée par les indicateurs de besoins de recherche perçus à partir des opinions des chefs d'entreprises agroalimentaires sur le climat des affaires et la protection de l'environnement. Cette approximation, de nature qualitative et subjective, peut s'avérer à la fois limitée et légitime dans un contexte où les données managériales objectives et stratégiques sur l'investissement innovant sont difficilement relevables. Le climat des affaires est perçu par le secteur entrepreneurial comme une contrainte majeure à l'activité des entreprises au Cameroun (Fotso et Tsafack, 2014). Les demandes d'amélioration de ce climat sont en permanence exprimées lors des Cameroon Business Forum $(C B F)$. En effet, le $C B F$, mis en œuvre depuis 2009, est constitué du Groupement Inter-patronal du Cameroun (GICAM), représentant le secteur privé, et des pouvoirs publics. Son objectif prioritaire est d'assurer l'attractivité de l'environnement juridique et fiscal de l'économie camerounaise.

La protection de l'environnement constitue également une variable importante, vu la croissance des contraintes liées aux normes que les produits camerounais (banane, bois, cacao, huile de palme..) rencontrent sur les marchés internationaux. Ces contraintes incitent ce secteur à investir sur ce thème par une demande d'innovations et de renforcement des capacités du personnel. Par ailleurs, certains travaux soulignent que la protection de l'environnement n'apparaît plus uniquement comme une contrainte à l'entreprise, mais comme variable génératrice de bénéfices sociaux et privés à moyen terme (Gallaud et al., 2012). De plus, d'autres travaux (Sarewitz et Pielke, 2007 ; Rafols et al., 2015) soulignent aussi la nécessité de concilier les préoccupations sociétales globales des entreprises et les préoccupations publiques nationales portant sur l'environnement et leurs adéquations avec l'offre de science.

Les indicateurs des besoins de recherche sont obtenus par le calcul, d'une part, des notes sur les obstacles au climat des affaires, et, d'autre part, des pourcentages des décisions d'investissement en matière de protection de l'environnement. Concernant les notes sur les obstacles au climat des affaires, on attribue les valeurs arbitraires $(3 ; 2,5 ; 2 ; 1,5$ et 1$)$ selon l'ordre décroissant avec lequel chaque responsable d'entreprise classe les cinq principaux facteurs (ou thématiques) de l'entrepreneuriat au Cameroun. La somme des notes pour chaque facteur mesure le niveau de priorité en matière de recherche. Par exemple, un facteur (obstacle) à note plus élevée exprime une demande de solution plus importante en matière de climat des affaires. Les pourcentages des décisions d'investissement pour la protection de l'environnement expriment, quant à eux, la part des entreprises souhaitant acquérir les technologies appropriées, les savoirs et savoir-faire pour préserver l'environnement des nuisances.

Nous testons donc:

- si le classement des obstacles du climat des affaires identifiés par les entreprises reflète le nombre d'apparitions du terme (pertinence) associé à chacun d'entre eux dans la littérature ;

- si le pourcentage d'entreprises ayant investi sur chaque variable environnementale reflète le nombre d'apparitions du terme (pertinence) associé à chacune d'entre elles dans la littérature. 
Une représentation graphique de l'offre et de la demande (besoins) des thématiques liées au climat des affaires et à la protection de l'environnement est proposée, avec en abscisses, les niveaux de pertinence offerts, d'une part, et en ordonnées, les indicateurs de besoins de recherche sur les thématiques, d'autre part. La droite à $45^{\circ}$, divisant le repère en deux parts égales, représente l'alignement parfait entre l'activité de recherche et les besoins des entreprises.

Les sources de données sont doubles. Premièrement, les données bibliométriques proviennent du Web of Science (WoS) et de Scopus. Elles mesurent l'activité de recherche au Cameroun en $E 2 A$ sur la période 1991-2015, avec la condition que l'un des auteurs soit affilié à un organisme basé au Cameroun et y dispose d'une adresse postale. Les requêtes d'interrogation des moteurs de recherche ont porté uniquement sur les articles et reviews (respectivement $95 \%$ et $5 \%$ des références), au regard des critères de pertinence, crédibilité et légitimité définissant l'utilité de l'information scientifique.

La requête (Encadré 1) s'étend particulièrement aux termes «food» et «nutrition». En effet ces deux termes focalisent principalement les investissements dans l'industrie agroalimentaire

Encadré. Requête pour la recherche documentaire dans WoS et Scopus.

La formule de requête est la suivante: Address or Affiliation country $=$ (Cameroon OR Cameroun) AND Year Published $=(1991-2015)$ AND Document Type $=$ (article OR review) AND Topic $=\left(\right.$ agricultur* ${ }^{*}$ OR agronomy OR food OR nutrition OR environment*). $\left.{ }^{*}\right)$ indique une troncature pour capturer tous les termes avec «agricultur...» ou « environment...».

On a ainsi généré 1214 et 1708 références bibliométriques respectivement du WoS Core Collection et de Scopus (sur un ensemble de publications de respectivement 9146 et 10557 ). Ce référencement concentre l'analyse sur les revues à comité de lecture scientifique et ignore l'offre de connaissances et d'informations que proposent les revues non-académiques ou les rapports de recherche.

L'échantillon bibliométrique constitué répertorie 33185 termes (thématiques). Pour éliminer les termes non pertinents, nous avons pré-identifié ceux qui satisfont le seuil d'apparition minimal de 5. Ainsi, 1537 termes ont été obtenus, et 1 'analyse de leur intérêt a conduit à en retenir seulement $80 \%$ (soit 1230). Les termes scientifiques relativement synonymes ou associés aux thématiques de besoins de recherche étudiés ont été regroupés sous l'appellation de ces dernières.

Deuxièmement, les données d'entreprises proviennent du tout premier recensement général des entreprises réalisé par l'Institut national de la statistique (INS) en 2008 au Cameroun. Trois cent dix-sept entreprises agroalimentaires formelles de différentes tailles ont été identifiées, représentant $20,4 \%$ de l'emploi du système productif du pays.

Le décalage entre les dates permet de prendre en compte les décalages de faisabilité scientifique. La faisabilité d'une recherche peut dépendre de la compétence et de l'engouement des chercheurs à mener certains travaux, des moyens de recherche mobilisables, de l'actualité, de la demande des utilisateurs finaux, etc. L'enquête «Entreprises» de 2008 rendue publique en 2010 a été réalisée pour combler des besoins en informations publiques sur les réalités de l'innovation dans les entreprises camerounaises. Elle se situe à une période où le nombre de publications a commencé à augmenter fortement au Cameroun.

\section{Le «non-alignement » de la production scientifique et des demandes du secteur entrepreneurial}

Le «non-alignement » (ou la non-concordance) apparaît en mettant en correspondance graphique les besoins des entreprises et l'offre scientifique.

Les besoins sur l'amélioration du climat des affaires et la protection de l'environnement sont recensés au tableau 2.

Pour le climat des affaires au Cameroun, les gestionnaires attribuent des notes relativement élevées pour fiscalité, corruption, formalités administratives et accès au crédit. Tandis qu'en matière de protection de l'environnement, il apparaît qu'à peine plus de $20 \%$ s'y intéressent en portant leurs décisions d'investissement essentiellement sur la gestion des déchets (non-radioactifs), le recyclage des eaux usées et la préservation de l'air et du climat.

Le tableau montre également l'importance des variables selon la taille de l'entreprise. Alors que la fiscalité l'emporte sur tous les autres facteurs quel que soit le type d'entreprise, il apparaît en revanche un engagement environnemental relativement plus marqué dans les grandes entreprises (GE), avec $50 \%$ d'entre elles plutôt engagées dans la gestion des déchets.

Considérant l'offre de recherche, il ressort que les publications en $E 2 A$ ont contribué pour $13 \%$ et $16 \%$ à l'offre de publication nationale, respectivement dans WoS et Scopus. Cependant, après une tendance haussière dès 1991, l'évolution du rapport des publications en E2A sur l'ensemble des publications (Fig. 1) indique une stabilité du pourcentage de 2011 à 2015, autour de $15 \%$, attribuable à l'essor parallèle des publications relevant d'autres disciplines scientifiques.

La correspondance entre les préoccupations environnementales ou sur le climat des affaires des firmes agroalimentaires, et l'indicateur de pertinence des thématiques abordées par les auteurs est limitée (Fig. 2 et 3).

Ainsi, si les déchets non radioactifs, eaux usées ou bruits et vibrations préoccupent un nombre relativement important d'entreprises, leur pertinence s'avère relativement faible dans la littérature scientifique camerounaise. Idem pour des thématiques à fortes notes en affaires (fiscalité, corruption, accès au crédit, formalités administratives, eau et énergie), quand bien même elles constituent des sujets abondamment traités dans les médias nationaux. Cette faible pertinence se confirme encore sur des facteurs contributeurs du processus d'innovation tels que formation, compétence, infrastructures, et opportunités de marché.

En revanche, une thématique comme sites, paysages et biodiversité a une pertinence de recherche relativement élevée, contrastant avec une utilité entrepreneuriale relativement faible. Néanmoins, des tentatives de convergence entre les chercheurs et les entreprises semblent s'établir sur les aspects 
Tableau 2. Obstacles au climat des affaires les plus cités et investissement sur la protection de l'environnement. Table 2. The most cited barriers to business climate and investment in environmental protection.

\begin{tabular}{|c|c|c|c|c|c|}
\hline Entreprises & $1^{\mathrm{er}}$ obstacle & $2^{\mathrm{e}}$ obstacle & $3^{\mathrm{e}}$ obstacle & $4^{\mathrm{e}}$ obstacle & $5^{\mathrm{e}}$ obstacle \\
\hline GE & Fiscalité & Infrastructures & $\begin{array}{l}\text { Corruption/formalités } \\
\text { administratives }\end{array}$ & Corruption & Formalités administratives \\
\hline ME & Fiscalité & Fiscalité & Corruption & Eau et énergie & Formalités administratives \\
\hline PE & Fiscalité & Fiscalité & Corruption & Concurrence déloyale & Formalités administratives \\
\hline TPE & Fiscalité & Coût du financement & Corruption & Fiscalité & Infrastructures \\
\hline
\end{tabular}

Pourcentage d'entreprises investissant sur la protection de l'environnement

\begin{tabular}{llllllll}
\hline & Nombre & Eaux usées & $\begin{array}{l}\text { Bruits et } \\
\text { vibrations }\end{array}$ & Déchets & $\begin{array}{l}\text { Sols et eaux } \\
\text { souterraines } \\
\text { et de surface }\end{array}$ & Air et climat & $\begin{array}{c}\text { Sites, paysage et } \\
\text { biodiversité }\end{array}$ \\
\hline $\mathrm{GE}$ & 38 & 36,8 & 18,4 & 50 & 31,6 & 31,6 & 29 \\
$\mathrm{ME}$ & 78 & 8,9 & 7,7 & 10,2 & 6,4 & 6,4 & 6,4 \\
$\mathrm{PE}$ & 91 & 11 & 3,3 & 11 & 1,1 & 5,5 & 1,1 \\
$\mathrm{TPE}$ & 110 & 24,5 & 2,7 & 26,4 & 1,8 & 22,7 & 1,8 \\
\hline
\end{tabular}

Source : RGE 2008. GE, ME, PE, TPE, respectivement pour grande, moyenne, petite et très petite entreprise. Nota Bene : au Cameroun, une TPE emploie au plus 5 personnes et a un chiffre d'affaires annuel hors taxes n'excédant pas 15 millions de francs CFA; une PE emploie entre 6 et 20 personnes et réalise un chiffre d'affaires compris entre 15 et 100 millions; une ME emploie entre 21 et 100 personnes avec un chiffre d'affaires compris entre 100 millions et un milliard; et GE emploie plus de 100 personnes et réalise plus d'un milliard de chiffre d'affaires (loi $\mathrm{n}^{\circ} 001 / 2010 \mathrm{du} 13$ avril 2010 portant promotion des petites et moyennes entreprises). Un euro $=655,957 \mathrm{FCFA}$.

\section{POURCENTAGE DE PUBLICATIONS}

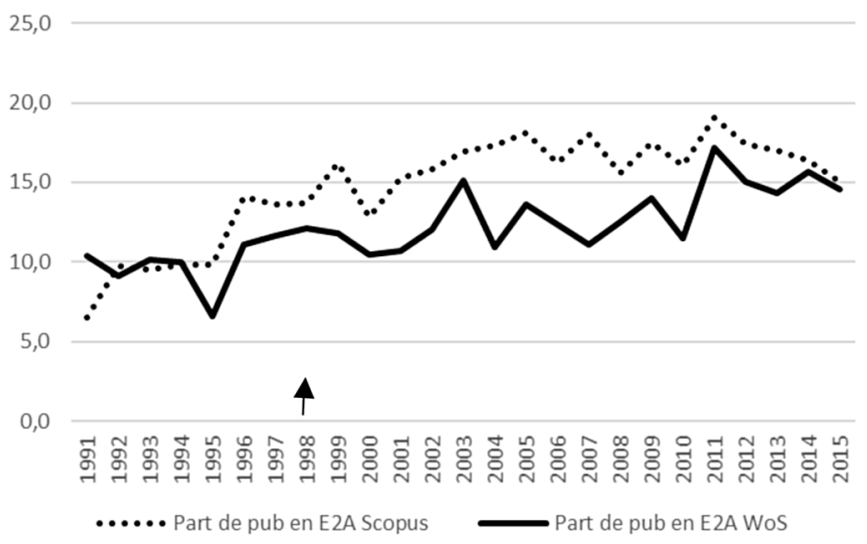

Fig. 1. Évolution relative des publications en $E 2 A$. Source: WoS et Scopus.

Fig. 1. Relative evolution of E2A publications.

concernant air et climat, puis sol et eaux souterraines et de surface.

Le manque de concordance entre l'offre et la demande de recherche questionne la gouvernance des priorités et thématiques de recherche mais également la capacité du secteur entrepreneurial à investir sur des technologies qui mobilisent mieux les ressources cognitives et techniques que propose la recherche. De manière concomitante, ce besoin de concordance interpelle aussi les structures qui produisent des informations et des statistiques sur l'innovation. En l'occurrence, l'enquête de l'INS explicite peu la demande de «recherche» des entreprises. Elle informe peu aussi sur la spécificité des activités de recherche et d'innovation du secteur entrepreneurial dans le contexte des pays en développement (Hadhri et al., 2016). Enfin, elle met en opacité la demande du secteur qualifié d'informel, dominé par des activités quasiartisanales (Kamdem et Schamp, 2014). Or, ce secteur concentre l'essentiel de l'emploi et porte des dynamiques d'investissement et d'innovation.

Notons que les résultats obtenus découlent d'un cadre méthodologique mobilisant des données secondaires produites et disponibles par et dans les pays en développement. Dans un contexte où l'émergence d'institutions régionales en Afrique pose l'investissement dans la recherche comme un levier des mécanismes de croissance et de développement (Clark et Frost, 2016), ce manque de «concordance » interpelle la recherche en sciences sociales pour évaluer les mécanismes qui explicitent la nature de ce levier (Temple et al., 2018). En l'occurrence, ce manque de concordance ne signifie pas que les industries agroalimentaires n'ont pas besoin de recherche scientifique, notamment dans les pays en développement où un besoin de valorisation des transformations agroalimentaires innovantes reflétant la «biodiversité alimentaire » s'exprime : savoir-faire locaux, diversité des ingrédients et produits utilisés, qui sont encore très mal connus (Fofiri et al., 2015).

\section{Conclusion}

L'utilité d'une recherche scientifique est un sujet controversé : selon certains auteurs tels que Polanyi (1962), l'offre de connaissance doit demeurer autonome et déconnectée de toute interférence externe ou demande. Les perspectives de retombées sociétales ne devraient donc pas, dans cette posture, guider la politique scientifique, en particulier pour les décisions d'allocation des fonds. En effet, l'utilité de la science est relative : elle dépend des contextes, de la période 


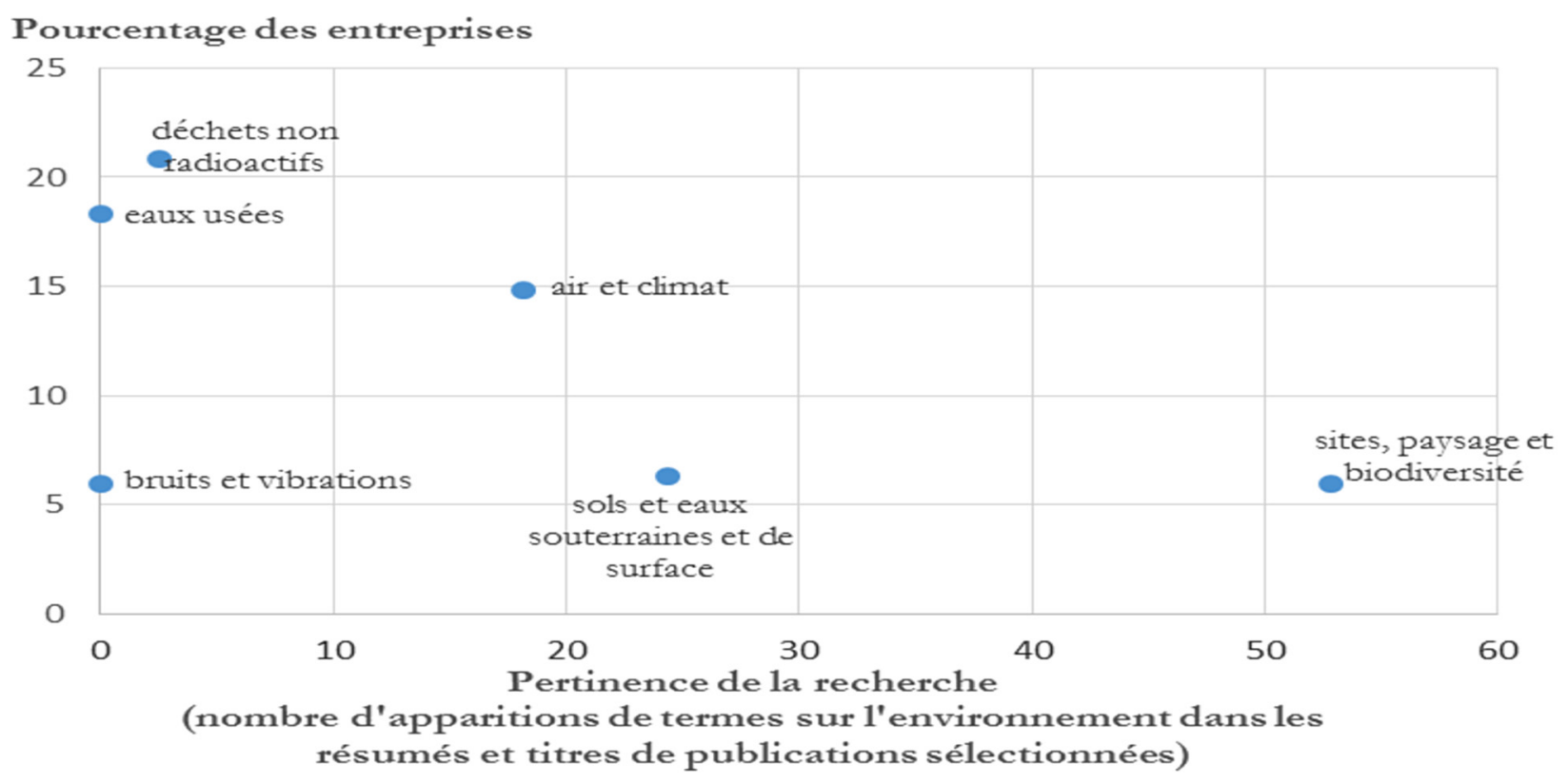

Fig. 2. Convergence entre offre et demande de recherche pour la protection de l'environnement. Source: Termes traduits de l'anglais à partir du WoS.

Fig. 2. Convergence between supply and demand for research for the protection of environment.

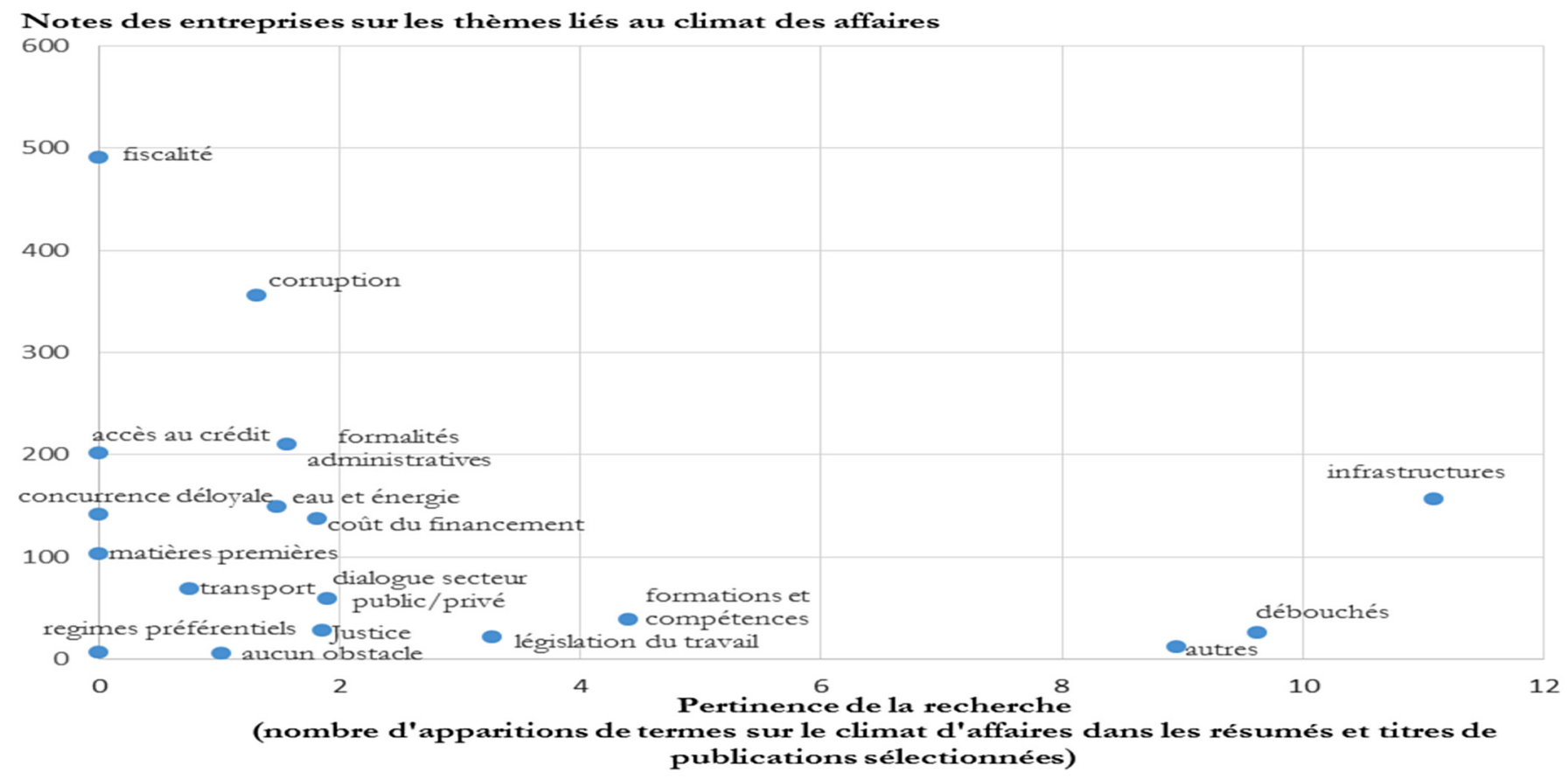

Fig. 3. Convergence entre offre et demande de recherche pour l'amélioration du climat des affaires.

Source: Termes traduits de l'anglais à partir du WoS.

Fig. 3. Convergence between supply and demand for research for improving business climate.

étudiée, de l'allocataire des fonds de recherche, des lobbys, du hasard. Les résultats sur l'adéquation entre l'offre et la demande de science sur le climat des affaires et la protection de l'environnement s'inscrivent dans cette relativité. Ainsi, les données bibliométriques utilisées comme indicateur de l'offre de recherche camerounaise, laissent à penser que cette dernière serait plus alignée sur la demande d'utilisateurs internationaux disposant de fonds de recherche, plutôt que sur celle, nationale, estimée à partir des opinions des gestionnaires d'entreprise du secteur productif. Certaines questions, par exemple, celle de la corruption, sont délicates à aborder du fait des biais d'observations. Cependant, les aspects relevant de la fiscalité 
ou de l'accès au crédit des entreprises constitueraient potentiellement des champs de recherche fertiles pouvant déboucher sur des recommandations fortes en termes de financement de l'innovation.

La faible correspondance entre les priorités des entreprises agroalimentaires et la production de connaissances scientifiques est mise en évidence par nos résultats. De fait, une contribution majeure de la recherche scientifique est restée non analysée au regard d'une démarche méthodologique focalisée sur l'usage d'une base de données bibliométriques et d'une enquête nationale sur les besoins du secteur entrepreneurial. Cette contribution concerne la formation de compétences et de capacités d'apprentissage, parfois rassemblées sous le vocable de "capital humain " (Arvanitis et al., 2016), qui sont aussi des sources majeures de processus d'innovation plus fondés sur l'imitation et le transfert de technologies que sur la conception d'innovations technologiques (Godin et Vinck, 2017).

Enfin, l'hypothèse méthodologique retenue pour assurer les critères de pertinence et de légitimité restreint l'analyse de l'offre de connaissances aux seules publications scientifiques. Cela écarte la production des revues sans comité de lecture et les rapports de recherche, qui constituent une part importante des publications des chercheurs du Sud. Résoudre ce problème pose un autre défi méthodologique, et nécessite donc d'autres travaux, pour sécuriser différemment les indicateurs de pertinence et de légitimité.

Le test d'applicabilité d'un cadre d'analyse méthodologique d'évaluation de la relation offre-demande de recherche dans le contexte des bases de données secondaires disponibles dans un pays en développement apporte des informations sectorielles mais ne peut être exhaustif sur l'analyse d'un alignement général. La mise en usage d'un cadre d'analyse des relations entre l'offre et la demande de recherche dans le contexte d'un pays en développement permet de caractériser l'insuffisance des bases d'informations qui pourraient ainsi être mieux référencées dans les futures enquêtes. La concordance entre l'offre et la demande de recherche en serait améliorée. L'accès à ces informations sur l'innovation serait aussi un apport important pour élaborer des politiques de recherche et d'innovation pertinentes.

Remerciements. Les auteurs remercient les évaluateurs anonymes pour leur contribution à l'amélioration de l'article.

\section{Références}

Alston JM. 2010. The benefits from agricultural research and development, innovation, and productivity growth. OECD Food, Agriculture and Fisheries Papers 31. DOI: https://doi.org/10.1787/ $5 \mathrm{~km} 91 \mathrm{nfsnkwg-en.}$

Arvanitis S, Seliger F, Stucki, T. 2016. The relative importance of human resource management practices for innovation. Economics of Innovation and New Technology 25(8): 769-800.

Arocena R, Sutz J. 2010. Weak knowledge demand in the South: learning divides and innovation policies. Science and Public Policy 37(8): 1-12.

Bisson P, Alami S, Temple L. 2014. Étude sur l'économie de la recherche au Cameroun. Programme d'Appui à la Recherche. Ministère de la recherche scientifique et de l'innovation. Yaoundé (Cameroun) : Cirad.
Brewer PR, Ley BL. 2013. Whose science do you believe? Explaining trust in source of scientific information about the environment. Science Communication 35(1): 115-137.

Casadella V, Tahi S. 2017. Capacités et politiques d'innovation dans les pays moins avancés: enseignements tirés du cas du Sénégal. Innovations 2: 13-39.

Clark N, Frost A. 2016. It's not STI: It's ITS - the role of science, technology and innovation (STI) in Africa's development strategy. International Journal of Technology Management \& Sustainable Development 15(1): 3-13.

Fofiri NEJF, Temple L, Ndjouenkeu R. 2015. La contribution de la recherche universitaire à la formation d'un système sectoriel d'innovation agroalimentaire au Cameroun. Innovations 47(2): 55-77.

Fotso A, Tsafack Nanfosso R. 2014. 100 propositions pour l'émergence du Cameroun. Yaoundé(Cameroun): Clé \& Gicam.

Gaillard J, Khelfaoui H. 2000. La science au Cameroun. In : Waast R, Gaillard J, eds. La Science en Afrique à l'aube du 21 siècle. Paris : Commission Européenne, DG XII, Rapport final, 547 p.

Gallaud D. 2014. Les systèmes nationaux d'innovation approches théoriques. In: Boutillier S, Forest J, Gallaud D, Laperche B, Tanguy C, Temri L, eds. Principes d'économie de l'innovation. Bruxelles: PIE Peter Lang, pp. 377-402.

Gallaud D, Martin M, Reboud S, Tanguy C. 2012. La relation entre innovation environnementale et réglementation : une application au secteur agroalimentaire français. Innovations 1(37): 155-175. DOI: $10.3917 /$ inno.037.0155.

Godin B, Vinck D. 2017. Critical studies of innovation. Cheltenham (UK): Edward Elgar, 335 p.

Gueutin C-A. 2012. L'essentiel de l'introduction à l'économie. Paris : Ellipses, 143 p.

Hadhri W, Arvanitis R, M'Henni H. 2016. Determinants of innovation activities in small and open economies: the Lebanese business sector. Journal of Innovation Economics \& Management 3: 77-107.

Jensen MB, Johnson B, Lorenz E, Lundvall BA. 2007. Forms of knowledge and mode of innovation. Research Policy 36(5): 680-693.

Kamdem SM, Schamp EW. 2014. L'université africaine et sa contribution au développement local : l'exemple du Cameroun. Paris : Karthala, 384 p.

Lundvall B. 1992. Relations entre utilisateurs et producteurs, systèmes nationaux d'innovation et d'internationalisation. In : Foray D, Freeman C, eds. Technologie et Richesse des Nations. Paris : Economica.

McNie E. 2007. Reconciling the supply of scientific information with user demands: an analysis of the problem and review of the literature. Environmental Science and Policy 10: 17-38.

Mouton J, Gaillard J, Milandré Van Lill M. 2015. Functions of science granting councils in Sub-Saharan Africa. In: Cloete N, Maasen P, Bailey T, eds. Knowledge production and contradictory functions in African higher education. Cape Town (RSA): African Minds, pp. $148-170$.

Rafols R, Ciarli T, Chavarro D. 2015. Under-reporting research relevant to local needs in the global south. Database biases in the representation of knowledge on rice. Proceeding Paper presented in the 2015 Globelics Conference, Havana, Cuba.

Reinert E. 2007. How rich countries got rich and why poor countries stay poor. London (UK): Constable, $400 \mathrm{p}$.

Rodrik D. 2007. One economics, many recipes: globalization, institutions, and economic growth. Princeton (NJ): Princeton University Press, 280 p.

Polanyi M. 1962. The republic of science: its political and economic theory. Minerva 1: 54-74.

Sarewitz D, Pielke Jr RA. 2007. The neglected heart of science policy: reconciling supply of and demand for science. Environmental Science and Policy 10: 5-16. 
Sutz J. 2012. Measuring innovation in developing countries: some suggestions to achieve more accurate and useful indicators. Int J Technological Learning, Innovation and Development 5(1/2): $40-57$.

Temple L, Machikou NN, Fongang FGF, Ndoumbè NM, Mathé S. 2017. Système national de recherche et d'innovation en Afrique: éclairage du Cameroun. Innovations 53: 41-67.
Temple L, Barret D, Blundo Canto G, Dabat MH, Devaux-Spatarakis A, Faure G, et al. 2018. Assessing impacts of agricultural research for development: a systemic model focusing on outcomes. Research Evaluation rvy005: 1-14. DOI: 10.1093/reseval/rvy005.

Tiran A, Uzunidis D. 2017. Dictionnaire économique de l'entrepreneur. Paris : Classiques Garnier, 374 p. https://halshs.archivesouvertes.fr/halshs-01491615.

Citation de l'article : Minkoua Nzié JR, Temple L. 2018. L'offre de recherche converge-t-elle vers les besoins du secteur agroalimentaire au Cameroun? Une analyse par la bibliographie. Cah. Agric. 27: 25008. 